\title{
Proportional fair buffer scheduling algorithm for 5G enhanced mobile broadband
}

 \\ 1,3,4,5,6 Laboratory of Artificial Intelligence, Data Sciences and Emerging Systems, Sidi Mohammed Ben Abdellah \\ University, Fez, Morocco \\ ${ }^{2}$ IMAGE laboratory, Moulay Ismail University, Meknes, Morocco
}

\begin{tabular}{|c|c|}
\hline Article Info & ABSTRACT \\
\hline Article history: & The impending next generation of mobile communications denoted $5 \mathrm{G}$ \\
\hline Received Aug 17, 2020 & intends to interconnect user equipment, things, vehicles, and cities. It will \\
\hline Revised Mar 26, 2021 & efficiency, and different combinations of use cases enhanced mobile \\
\hline Accepted Apr 11, 2021 & $\begin{array}{l}\text { broadband (eMBB), ultra reliable low latency communications (URLLC), } \\
\text { massive internet of things (mIoT) with new capabilities and diverse }\end{array}$ \\
\hline Keywords: & $\begin{array}{l}\text { requirements. Adoption of advanced radio resource management procedures } \\
\text { such as packet scheduling algorithms is necessary to distribute radio }\end{array}$ \\
\hline $5 \mathrm{G}$ & resources among different users efficiently. The proportional fair (PF) \\
\hline Downlink & scheduling algorithm and its modified versions have proved to be the \\
\hline eMBB & $\begin{array}{l}\text { commonly used scheduling algorithms for their ability to provide a tradeoff } \\
\text { between throughput and fairness. In this article, the buffer status is combined }\end{array}$ \\
\hline Resource allocation & with the PF metric to suggest a new scheduling algorithm for efficient \\
\hline Scheduling algorithm & $\begin{array}{l}\text { support for eMBB. The effectiveness of the proposed scheduling strategy is } \\
\text { proved through à comprehensive experimental analysis based on the } \\
\text { evaluation of different quality of service key performance indicators (QoS } \\
\text { KPIs) such as throughput, fairness, and buffer status. }\end{array}$ \\
\hline
\end{tabular}

This is an open access article under the CC BY-SA license.

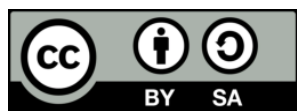

\section{Corresponding Author:}

Asmae Mamane

Laboratory of Artificial Intelligence

Data Sciences and Emerging Systems

Sidi Mohammed Ben Abdellah University

Imouzzer Road BP 2626 Fez 30000, Morocco

Email: asmae.mamane@gmail.com

\section{INTRODUCTION}

Based on analog technology, the first generation of mobile communications $1 \mathrm{G}$ was intended exclusively for voice communication. In the context of its evolution, $2 \mathrm{G}$ has improved voice and text messaging thanks to the digitization. The third-generation $3 \mathrm{G}$ was developed to support optimized voice as well as low-cost and low-data mobile Internet. By the time, the demand for high data application has increased, introducing the fourth generation $4 \mathrm{G}$ known as long term evolution (LTE) and offering high capacity and data for large telecommunications networks. Aiming to enable a fully connected world, 5G communication incorporates current and new infrastructures, innovative and heterogeneous technologies to satisfy the severe capability needs and guarantee maximum coverage for users in different circumstances [1]. This full connectivity is targeted not only to communicating people, but also enables the communication of machines and things that can bring evaluated value to improve the operational efficiency of the society and facilitate our everyday life [2]. 
Towards this achievement, three main use scenarios are supported by $5 \mathrm{G}$. According to international telecommunications union-radio communication sector (ITU-R), 5G use cases are classified as enhanced mobile broadband (eMBB) requiring higher data rates, ultra-reliable and low latency communications (URLLC) where devices rely on very short latency to perform their task and massive machine-type communications that connect a large number of devices [3]-[5]. To support these use cases, new requirements have been defined such as $10 \mathrm{Gbps}$ throughput for eMBB, a latency of 1 millisecond for URLLC cases, and a high connection density for massive machine type communications (mMTC) [6]. In addition to these specifications, the upcoming $5 \mathrm{G}$ technology proposes common requirements for all use cases, it aims to increase bandwidth efficiency, improve energy consumption, and provide a scalable and customizable network [7]. Despite the benefits of the physical layer features that come in support of those requirements, improving the spectral efficiency and increasing the throughput to meet the quality of service (QoS) requirements of eMBB remains challenging [8].

In the literature, numerous articles considered radio resource allocation, and proposed divers scheduling algorithms and schemes. As presented in [9], C. F. Müller the Round Robin algorithm consists of sharing the resources equally to serve all user equipments (UEs) in the current cell. Known as one of the simplest scheduling algorithms, Round Robin is used by many systems because it is easy to implement. The sest CQI scheme takes the channel quality information into account, by assigning resource blocks to users with the best radio link conditions through the analysis of UE feedback that contains channel quality indicator (CQI) as detailed in [10]. The PF scheduler presented in [11] allocates resources to users according to their average achievable data rate, in this manner, it equally serves users even those having a very low CQI value. maximum largest weight delay first is a scheduling scheme for real-time and non-real-time services [12]; it considers the time of the first package of the queue $\mathrm{D}_{\mathrm{HOL} \text {, i }}$ to offer priority to real-time traffic. Exponential/PF (EXP/PF) considers both real time and non-RT traffic [13]. EXP-MLWDF, based on the modified largest weighted delay first (MLWDF) algorithm, this scheme promotes the users with bad channel conditions by applying the exponential term on the MLWDF metric as detailed in [14]. In [15], M. I. Saglam and M. Kartal the authors proposed a 5G NR eMBB downlink lean algorithm that aims to balance resource efficiency and flow fluency. In order to improve the overall QoS, the authors in [16] proposed a powerefficient QoS Scheduler. M. Kalil [17] proposes an efficient low-complexity scheduler that meets the scheduling to the virtualization techniques. The virtual token scheme (VT-MLWDF), Queue-HOL-MLWDF, Channel-QoS aware, and E-MQS schemes given in [18]-[21] respectively have associated virtual token queue to their metrics to avoid packet loss. The qos class identifier (QCI) scheduler presented in [22] consists on a scheduling algorithm based on the demands of QoS.

In this article, we focus on downlink traffic of eMBB, whose challenges reside in enhancing the user's achieved bit rate, reducing the amount of data in the buffer, and the limited available radio resources to satisfy these needs. Additionally, this paper is addressing the scheduling problem by dynamically assigning radio resource blocks (RBs) to each user according to its buffer status. As well as, a novel downlink resource allocation algorithm that uses the buffer status and channel conditions to address performance degradation is proposed. The new algorithm metric provides radio resource allocation to users based on the status of their buffer to satisfy the demands of QoS.

The rest of this paper is organized is being as, in section 2 we present the $5 \mathrm{G}$ technology architectures and its downlink scheduling process as defined by the 3GPP for release 15. Section 3 defines our novel scheduling algorithm. In section 4 we analyze and compare the performances of the newly developed algorithm with the literature scheduling schemes. Finally, a conclusion to summarize this work in section 5 .

\section{PROPOSED SCHEDULING ALGORITHM}

In the previous works [19], [21], [23], the buffer size was used only to avoid buffer overflow in order to minimize packet loss. Meanwhile, in our proposed algorithm we are using the buffer size information to perform an effective downlink resource allocation. The buffer status report is a signal sent from the UE to the nbridge (Enb) (called gNodeB (gNB) for $5 \mathrm{G}$ systems), carrying the information on how much data is in the buffer to be sent out. Since the buffer state information is tightly connected to the QoS, in our new scheduling algorithm called "The Proportional Fair Buffer", we use the buffer size parameter to allow a flexible assignment of the resource according to the amount of data to be sent. In this way, the flow with a higher buffer size will have a higher metric, hence the highest priority to have more RB assigned than other flows, and this will automatically enhance the achievable throughput. However, higher data rates are not the only requirements of eMBB, guarantee fairness between all cell users remains important. Thus, to maintain the network equity in our scheduling scheme, the second part of the metric is the same as the PF metric known to be a fair scheduler. Our metric expressed in is denoted $w_{i, j}$ to refer to the $\mathrm{i}$-th user at $\mathrm{j}$-th $\mathrm{RB}$.

Int J Elec \& Comp Eng, Vol. 11, No. 5, October 2021 : 4165 - 4173 


$$
w_{i, j}=\frac{r_{i, j}}{\overline{R_{i}}} \times b_{i}
$$

This metric is calculated by adding the buffer size denoted $b_{i}$, to the ratio of the instantaneous rate assigned to $i$-th flow at the $j$-th $R B r_{i, j}$, and the average transmission data rate $\bar{R}_{i}$. In the metric, the average throughput information is updated as in [24]. $\alpha$ parameter takes a value between 0 and 1. Each resource block is assigned to the subscribers with the highest metric order in each TTI.

$$
\overline{\mathrm{R}_{\mathrm{i}}}=\left((1-\alpha)^{*} \text { PastDataRate }\right)+\left(\alpha^{*} \text { InstantaneousDataRate }\right)
$$

The complete procedure of our new algorithm is summarized is being as:

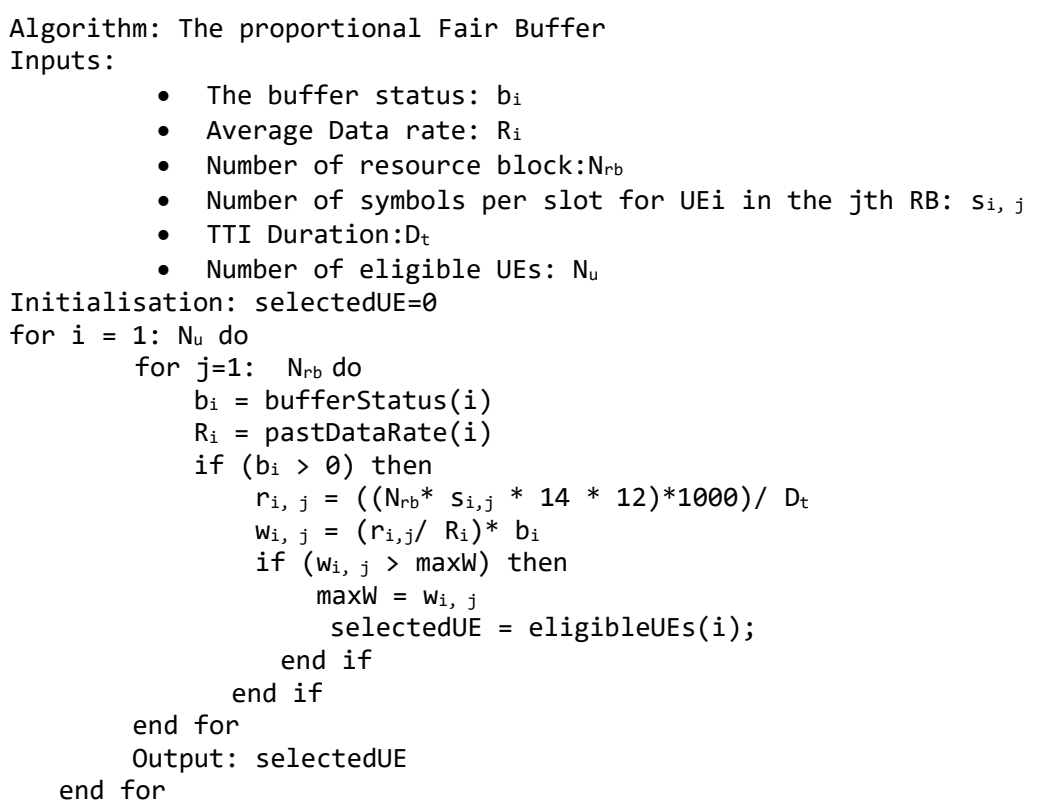

\section{RESEARCH METHOD}

The technical report European telecommunications standards institute (ETSI) 3 generation partnership project (3GPP) technical report (TR) 38.912 version 15.0.0 release 15 [25], presents two ranges of carrier frequencies, frequency range 1 denoted FR1 where frequency bands are sub-6GHz and frequency range 2 denoted FR2 which uses mm-Wave (beyond $6 \mathrm{GHz}$ ). The $5 \mathrm{G}$ new radio (NR) is expected to take advantage of this spectrum, with up to 400 megahertz of bandwidth and possibly more later. Moving onto more detailed points on the physical layer, the subcarrier spacing fixed in long term evolution (LTE) at 15 kilohertz can now take values between 15 and 240 kilohertz according to different numerologies specified in [26].

The study of non-standalone (NSA) between frequencies sub- $6 \mathrm{GHz}$ band and the mm-wave band was intensively conducted and concluded that for NSA both bands (used for LTE and NR) are below 6 GHz. [25] In this paper, our scheduling strategy, based on the non-standalone architecture, assigns the physical downlink shared channel (PDSCH) [27] resources to a set of static user equipment (UEs) connected to a gNB. In this simulation, we use frequency division duplexing (FDD) mode because frequency domain scheduling provides a significant contribution to the performance advancements. The performance of the scheduling strategy is evaluated in terms of achieved medium access control (MAC) throughput and fairness in resource sharing.

According to Technical Report 38.913, possible deployment scenarios are indoor, rural, with urban cells, air-to-ground, and connected cars as potential applications. In this work, we mainly concentrate on the resource scheduling for a downlink wireless network, where a single gNB is deployed at the center of an urban cell coverage area. High traffic loads and high user densities in city centers are the main features of the dense urban microcellular scenario [28]. Conforming to the attributes listed in Table 6.1.2-1 of the 3 GPPP technical report, the carrier frequency used for evaluation purposes of frequency range 1 (FR1) is 4 GHz, a 
density of 10 users per TRxP with full buffer traffic is the baseline for this scenario, also a max bandwidth of $100 \mathrm{MHz}$ is defined with $30 \mathrm{KHz}$ of subcarrier spacing, which results in $273 \mathrm{RB}$ as per 3 GPP TS 38.104 [29]. The sum-up of the main scenario parameters is presented in Table 1.

Table 1. Scenario parameters

\begin{tabular}{cc}
\hline Parameter & Value \\
\hline Carrier frequency & $4 \mathrm{GHz}$ macro layer \\
OFDMA Cyclic Prefix & Normal (7) [30] \\
Bandwidth & $100 \mathrm{MHz}$ \\
Number of slots/frames & 20 \\
Number of frames/simulations & 100 \\
Durations of frame & $10 \mathrm{~ms}$ \\
Simulation time & $1 \mathrm{~s}$ \\
Number of RB & 273 \\
Subcarrier spacing & $30 \mathrm{kHz}$ \\
Number of users & 10 \\
Number of cells & 1 \\
Modulation order & 64QAM -256QAM (depending on CQI value) \\
Channel Model Speed of UE & Pedestrian \\
Antenna number & 8 \\
Number of UE receive antennas & 2 \\
\hline
\end{tabular}

The performance evaluation of the proposed proportional fair buffer (PF-Buffer) downlink scheduler and other commercial schedulers is analyzed according to their throughput, goodput, fairness, and accumulated buffer values. The user performance corresponds to the ratio of the successfully delivered data bits by the overall simulation time [31]. The throughput is defined as an important indicator of the performance and quality of a network connection.

$$
\text { Throughput }=\frac{\sum \text { RxPacketSize }}{\text { DeliveryTime }}
$$

Goodput is an important performance measure to evaluate the effective rate experienced by the user. Also known as the application-level throughput of communication, the goodput considers the number of useful data bits successfully delivered over a communication channel, which is divided by the time it takes to successfully transmit it [32]. According to [33] the goodput is expressed by (4):

$$
\text { Goodput }=\frac{\text { UsefulData }}{\text { TransmissionTime }}
$$

Fairness in mobile systems has been widely studied as an important system performance metric enabling the equal sharing of radio resources between all the users [34]. A fairness calculation is necessarily correlated with a certain achievement, including reducing delays and maximizing the amount of resources allocated [35]. Fairness can be obtained in many ways considering different perspectives, the commonly used fairness metrics in the literature are detailed in [36]. According to the most used fairness metric, Jain's index [37], the level of equity received by each stream is the flow rate reached by each flow at the end of each simulation. In the (5), xi is the user's throughput, while $\mathrm{n}$ represents the active flows.

$$
\text { Fairness }_{\text {Index }}=\frac{\left(\sum x_{i}\right)^{2}}{n \times \sum x_{i}^{2}}
$$

\section{RESULTS AND DISCUSSION}

We present in this paragraph the analysis of the simulation results of the proposed proportional fair buffer (PFB) scheduler compared to conventional scheduling algorithms (Round Robin [9], best CQI [10], and proportional fair [11]) and the recent algorithms in the literature (CQI scheduler [22] and Lean Scheduler [15]). The obtained results are analyzed in terms of throughput, goodput, fairness, and accumulated buffer values.

Int J Elec \& Comp Eng, Vol. 11, No. 5, October 2021 : 4165 - 4173 


\subsection{Throughput}

Figure 1 shows that the best continuous quality improvement (CQI) and the proposed PF-Buffer provide high throughput in the order of 239.4 Mbps and $238 \mathrm{Mbps}$ respectively while the lean scheduler that provides 224.3 Mbps comes third and the QoS class identifier (QCI) scheduler takes the fourth position giving a $218 \mathrm{Mbps}$ throughput compared to the $\mathrm{PF}$ with 213.4 Mbps data rate and revenue regulations (RR) which does not exceed $200 \mathrm{Mbps}$. Despite providing the higher data rate value, the best CQI assigns only $0.23 \mathrm{Mbps}$ to users with bad channel conditions since its metric is based only on channel quality indicator. Whereas, the proposed algorithm guarantees to users having bad channel conditions and a higher amount of data to be sent in the buffer, a minimum of 9.2 Mbps throughput, surpassing significantly the minimum data rate of the other scheduling algorithms which does not exceed 4.6 Mbps. Furthermore, it can be seen from Figure 1 that the Best CQI max user data rate is higher than the proposed PF-Buffer algorithm, presenting respectively 59.9 Mbps and 56.4 Mbps. Meanwhile, the proposed algorithm is outperforming the Best CQI algorithm in terms of the gap between the maximum throughput and the minimum data rate that a user can have. Namely, the Best CQI and the proposed PFB algorithm present a gap of $59 \mathrm{Mbps}$ and $47.2 \mathrm{Mbps}$ respectively. Note that the gap information provides a clear idea about how efficiently the resources are allocated. Overall, the proposed algorithm seems to provide the best performance in terms of throughput.

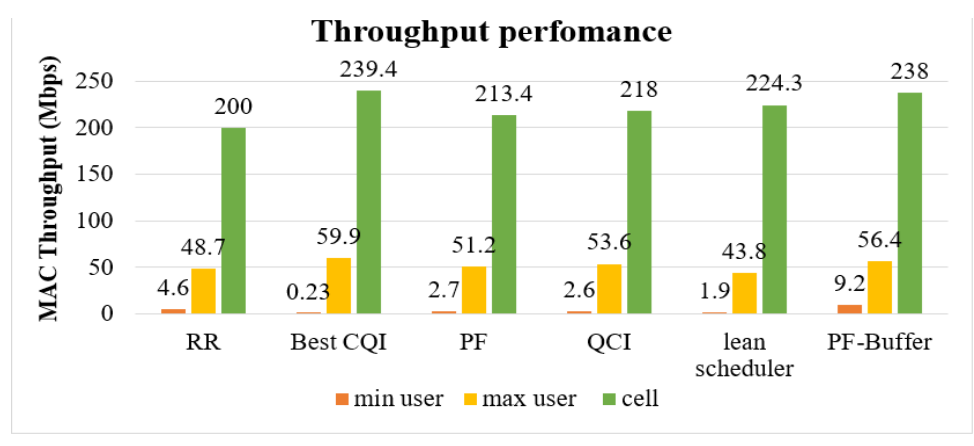

Figure 1. Throughput performance

\subsection{Goodput}

As depicted in Figure 2, the best CQI scheduler presents the higher cell goodput too, in a range of 214.4 Mbps, followed up by the proposed PF-Buffer with $212 \mathrm{Mbps}$. The lean scheduler comes third presenting a goodput of $200.7 \mathrm{Mbps}$. The QCI scheduler and PF provide almost the same goodput 197.8 Mbps and 195.5 Mbps respectively. The RR allocates the lowest goodput level in order of $173 \mathrm{Mbps}$ per cell. The proposed scheduling scheme guarantees a minimum goodput of $6.6 \mathrm{Mbps}$ for the distant users or those having poor channel conditions meanwhile the best CQI assigns only $0.1 \mathrm{Mbps}$ goodput for those users. As the goodput refers to the throughput achieved by the end application, a user having a higher goodput value means that this user is experiencing a high quality of experience (QoE) since he uses his application as much efficient as it is possible. According to the simulation results in Figure 2, the proposed PF-Buffer scheduling scheme affords the second highest cell goodput value to the users, besides, it is the only one that guarantees a meaningful goodput to cell edge users.

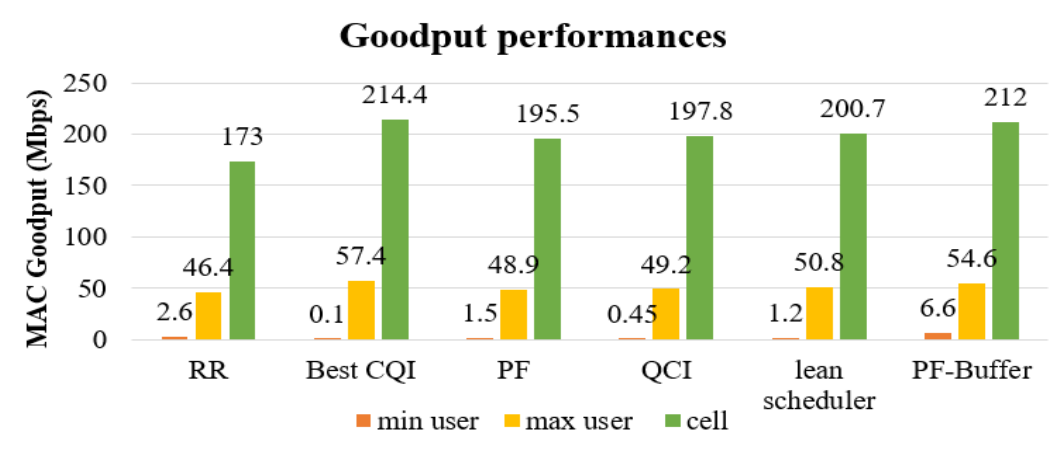

Figure 2. Goodput performance 


\subsection{Fairness}

It can be noticed from Figure 3, that fairness performance differs from a scheduler to another. Where the RR is the most equal algorithm having a fairness index that exceeds $98 \%$. The proposed PF-Buffer comes second with $91.28 \%$ fairness of resources sharing among the users. The QCI scheduler and the PF achieve respectively $86 \%$ and $84.11 \%$ besides the lean scheduler reach the $78.6 \%$ threshold while the best CQI achieved only $50 \%$ equity. As a result, our proposed scheme remains the fairest among these scheduling algorithms, following the Round Robin scheduler, known as the classical fair scheduling scheme in literature.

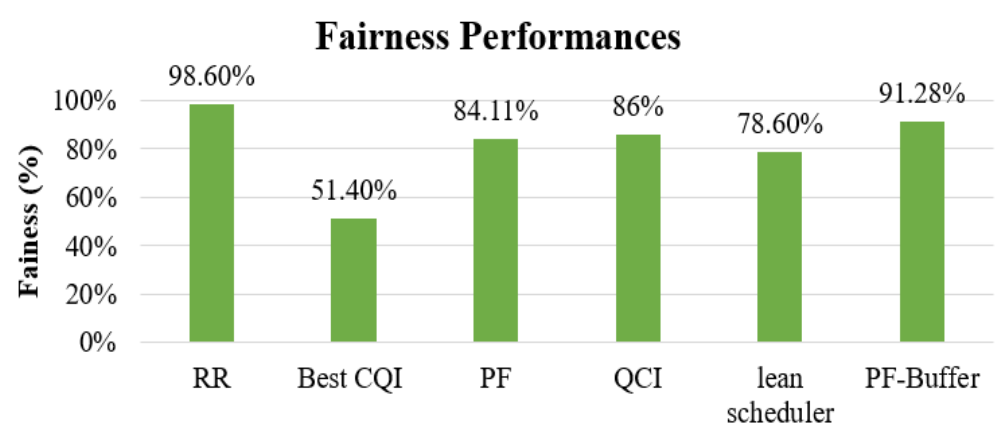

Figure 3. Fairness performance

\subsection{Buffer status}

The buffer status is an important measure of the performance of communication systems since it refers to the ability of networks to deliver all the data that one user transmits. In this simulation results, we analyze the accumulated buffer which is calculated by amassing the buffer status of all cell users at the end of the simulation. Remarkably, the proposed scheduling scheme enables to process the maximum data compared to the other algorithms. As illustrated in Figure 4, the proposed PF-Buffer algorithm permits the transmission of a big amount of data and empty the users' buffer, the accumulated buffer of users at the end of the evaluation simulation is $176.72 \mathrm{KBs}$, followed by the Best CQI scheme with $177 \mathrm{KBs}$ buffer data, whereas the lean scheduler and the PF scheduler buffer remain full of almost $228.8 \mathrm{KBs}$ and $244 \mathrm{KBs}$ respectively. The QCI scheduler and RR come in the last place with $255.2 \mathrm{KBs}$ and $300.72 \mathrm{KBs}$ respectively. Besides using the buffer as a parameter in our metric to enhance the overall scheduling performance, the proposed PFB algorithm avoids the buffer's overflow, therefore, it reduces the packets' loss and strengthens the reliability of the system that is an important requirement for $5 \mathrm{G} \mathrm{eMBB}$.



Figure 4. Accumulated buffer performance

As depicted in Table 2, the overall performance evaluation results show that the new scheduler (PF-Buffer) has a superior cell throughput and goodput performance over all the evaluated algorithms except the Best CQI. The fairness performance of our proportional fair buffer scheduler is located between RR and QCI scheduler. The novel scheduling scheme empties the users' buffer by transmitting the maximum data within the simulation duration. As stated previously, our proposed scheduling algorithm responds efficiently to $5 \mathrm{G}$ eMBB needs by providing the best tradeoff between the different requirements such as throughput, goodput, fair resource allocation, and reliability of the data transmitted. 
Table 1. Ranking of the evaluated methods

\begin{tabular}{ccccc}
\hline Rank & Throughput & Goodput & Accumulated buffer & Fairness \\
\hline 1 & Best CQI & Best CQI & PF-Buffer & RR \\
2 & PF-Buffer & PF-Buffer & Best CQI & PF-Buffer \\
3 & lean scheduler & lean scheduler & lean scheduler & QCI \\
4 & QCI & QCI & PF & PF \\
5 & PF & PF & QCI & lean scheduler \\
6 & RR & RR & RR & Best CQI \\
\hline
\end{tabular}

\subsection{Implementation and execution}

The presented experiments have been performed on a Lenovo LEGION with Intel ${ }^{\circledR}$ Core i5-9th Processor $2.4 \mathrm{GHz}$ Boost, $8 \mathrm{~GB}$ of RAM, running with Windows $10 x 64$ operating system and equipped with NVIDIA GeForce GPU GTX1660Ti. The proposed algorithm is coded using the 5G Toolbox of Matlab2020a environment. The authors are willing to share more details of this algorithm's steps and codes with the scientific community.

\section{CONCLUSION}

The enhanced Mobile BroadBand (eMBB) of the fifth-generation wireless systems aims to provide higher performances compared to its anterior $4 \mathrm{G}$ besides assigning radio resources efficiently. In this perspective, several works have studied radio resource allocation and scheduling. Throughout this paper, we optimized 5G mobile communication resources and flow efficiencies by exploiting the buffer status parameter. As a result, we propose a new downlink scheduling algorithm designed for eMBB. The proposed scheduler aims to provide an efficient downlink scheduling of UEs. The metric uses the channel condition information and the buffer status to allocate radio resources to the users. Compared to classic schedulers and newly developed algorithms in the literature, the new scheme is more accurate according to efficiency, fairness, and buffer status. Our proposed scheduling algorithm provides a good tradeoff between the fairness and the experienced data rate by guaranteeing a minimum acceptable goodput to all the users in the cell. Also, it enables a fluent transmission of the buffer's data. Despite the enhancement provided by our new scheduling algorithm, we intend in future works to propose a new scheduling algorithm that increases the experienced data rate to meet the requirements of eMBB $5 \mathrm{G}$ networks for dense urban scenarios.

\section{ACKNOWLEDGMENTS}

This work is carried out with the support of the National Center for Scientific and Technical Research (CNRST) through the Research Excellence Scholarships Program.

\section{REFERENCES}

[1] A. Mamane, M. El Ghazi, G. R. Barb, and M. Otesteanu, "5G Heterogeneous Networks: An Overview on Radio Resource Management Scheduling Schemes," 2019 7th Mediterranean Congress of Telecommunications (CMT), Fez, Morocco, 2019, pp. 1-5, doi: 10.1109/CMT.2019.8931369.

[2] S. Kumar, T. Agrawal, and P. Singh, "A Future Communication Technology : 5G," International Journal of Future Generation Communication and Networking, vol. 9, no. 1, pp. 303-310, 2016, doi: 10.14257/ijfgcn.2016.9.1.26.

[3] A. MAMANE, M. El Ghazi, S. Mazer, M. El Bekkali, M. Fattah, and M. Mahfoudi, "The impact of scheduling algorithms for real-time traffic in the 5G femto-cells network," 2018 9th International Symposium on Signal, Image, Video and Communications (ISIVC), pp. 147-151, 2018, doi: 10.1109/isivc.2018.8709175.

[4] A. Es-Saqy, M. Abata, M. Mehdi, S. Mazer, M. Fattah, M. El Bekkali et al., "28 GHz balanced pHEMT VCO with low-phase noise and high output power performance for $5 \mathrm{G} \mathrm{mm-wave} \mathrm{systems,"} \mathrm{International} \mathrm{Journal} \mathrm{of} \mathrm{Electrical}$ and Computer Engineering (IJECE), vol. 10, no. 5, pp. 4623-4630, 2020, doi: 10.11591/ijece.v10i5.pp4623-4630.

[5] A. Es-Saqy, M. Abata, M. Mehdi, S. Mazer, M. Fattah, M. El Bekkali et al., "A 5G mm-wave compact voltagecontrolled oscillator in $0.25 \mu \mathrm{m}$ pHEMT technology," International Journal of Electrical and Computer Engineering (IJECE), vol. 11, no. 2, pp. 1036-1042, 2021, doi: 10.11591/ijece.v11i2.pp\%25p.

[6] M. A. Siddiqi, H. Yu, and J. Joung, "5G ultra-reliable low-latency communication implementation challenges and operational issues with IoT devices," Electronics (Switzerland), vol. 8, no. 9. pp. 1-18, 2019, Art. No. 981, doi: 10.3390/electronics8090981.

[7] B. S. Chaudhari, M. Zennaro, and S. Borkar, "LPWAN technologies: Emerging application characteristics, requirements, and design considerations," Future Internet, vol. 12, no. 3, pp. 1-25, 2020, Art. No. 46, doi: 10.3390/fi12030046.

[8] B. Younes, F. Mohammed, M. Saï, and M. El Bekkali, "5G uplink interference simulations, analysis and solutions: the case of pico cells dense deployment," International Journal of Electrical and Computer Engineering (IJECE), vol. 11, no. 3, pp. 2245-2255, Jun. 2021, doi: 10.11591/ijece.v11i3.pp2245-2255.

[9] C. F. Müller, G. Galaviz, Á. G. Andrade, I. Kaiser, and W. Fengler, "Evaluation of Scheduling Algorithms for 5G 
Mobile Systems," in Studies in Systems, Decision and Control, vol. 143, Springer International Publishing, 2018, pp. 213-233, doi: 10.1007/978-3-319-74060-7_12.

[10] A. Hajjawi et al., "Investigation of the Impact of Different Scheduling Algorithm for Macro-Femto-Cells over LTE-A Networks," 2016 IEEE 3rd International Symposium on Telecommunication Technologies (ISTT), pp. 28-30, 2016, doi: 10.1109/ISTT.2016.7918098.

[11] A. Biernacki and K. Tutschku, "Comparative performance study of LTE downlink schedulers," Wireless personal communications, vol. 74, no. 2, pp. 585-599, Jan. 2014, doi: 10.1007/s11277-013-1308-4.

[12] P. Ameigeiras, J. Wigard, and P. Mogensen, "Performance of the M-LWDF scheduling algorithm for streaming services in HSDPA," IEEE 60th Vehicular Technology Conference, 2004. VTC2004-Fall. vol. 2, 2004, pp. 9991003, doi: 10.1109/vetecf.2004.1400171.

[13] S. Dardouri and R. Bouallegue, "Comparative Study of Scheduling Algorithms for LTE Networks," Wireless Personal Communications, vol. 82, no. 3, pp. 1405-1418, 2015, doi: 10.1007/s11277-015-2289-2.

[14] M. Mahfoudi, M. El Bekkali, A. Najd, M. El Ghazi, and S. Mazer, "A New Downlink Scheduling Algorithm Proposed for Real Time Traffic in LTE System," International Journal of Electronics and Telecommunications, vol. 61, no. 4, pp. 409-414, 2015, doi: 10.2478/eletel-2015-0054.

[15] M. I. Saglam and M. Kartal, "5G Enhanced Mobile Broadband Downlink Scheduler," In 2019 11th International Conference on Electrical and Electronics Engineering (ELECO), 2019, pp. 687-692, doi: 10.23919/ELECO47770.2019.8990378.

[16] M. Kalil, A. Shami, and A. Al-Dweik, "Power-efficient QoS scheduler for LTE uplink," IEEE International Conference on Communications (ICC), pp. 6200-6204, 2013, doi: 10.1109/ICC.2013.6655598.

[17] M. Kalil, et al., "Efficient Low-Complexity Scheduler for Wireless Resource Virtualization," IEEE Wireless Communications Letters, vol. 5, no. 1, pp. 56-59, 2016, doi: 10.1109/LWC.2015.2495206.

[18] M. Iturralde, T. A. Yahiya, A. Wei, and A.-L. Beylot, "Performance Study of Multimedia Services Using Virtual Token Mechanism for Resource Allocation in LTE Networks," 2011 IEEE Vehicular Technology Conference (VTC Fall), 2011, pp. 1-5, doi: 10.1109/VETECF.2011.6092905.

[19] M. M. Nasralla and M. G. Martini, "A downlink scheduling approach for balancing QoS in LTE wireless networks," 2013 IEEE 24th Annual International Symposium on Personal, Indoor, and Mobile Radio Communications (PIMRC), pp. 1571-1575, 2013, doi: 10.1109/PIMRC.2013.6666392.

[20] D. Samia and B. Ridha, "A New Scheduling Algorithm for Real-Time Communication in LTE Networks," In 2015 IEEE 29th International Conference on Advanced Information Networking and Applications Workshops, 2015, pp. 267-271, doi: 10.1109/WAINA.2015.36.

[21] D. H. Nguyen, H. Nguyen, and E. Renault, "A new Channel-and QoS-Aware Scheduling Scheme for Real-time Services in LTE Network," International Journal of Applied Information Systems, vol. 11, no. 4, pp. 1-8, 2016, doi: 10.5120/ijais2016451601.

[22] M. Mamman, Z. M. Hanapi, A. Abdullah, and A. Muhammed, "Quality of service class identifier (QCI) radio resource allocation algorithm for LTE downlink," PLoS One, vol. 14, no. 1, pp. 1-22, Jan. 2019, doi: 10.1371/journal.pone.0210310.

[23] D. H. Nguyen, H. Nguyen, and É. Renault, "E-MQS - A new downlink scheduler for real-time flows in LTE network," In 2016 IEEE 84th Vehicular Technology Conference (VTC-Fall), 2017, pp. 1-5, doi: 10.1109/VTCFall.2016.7880981.

[24] Z. Sun, C. Yin, and G. Yue, "Reduced-complexity proportional fair scheduling for OFDMA systems," 2006 International Conference on Communications, Circuits and Systems, vol. 2, 2006, pp. 1221-1225, doi: 10.1109/ICCCAS.2006.284866.

[25] 3GPP TR 38.912, “5G; Study on New Radio (NR) access technology," ETSI TR 138912 V15.0.0, pp. 1-76, 2018.

[26] 3GPP, "TS 138 211-V15.7.0- "5G; NR; Physical channels and modulation (3GPP TS 38.211 version 15.7.0 Release 15)," ETSI TR 138912 V15.0.0, pp. 1-99, 2019.

[27] A. Maroua and F. Mohammed, "Characterization of Ultra Wide Band indoor propagation," 2019 7th Mediterranean Congress of Telecommunications (CMT), 2019, pp. 1-4, doi: 10.1109/CMT.2019.8931367.

[28] 3GPP TR 38.913:, "5G; Study on scenarios and requirements for next generation access technologies," Etsi $\operatorname{Tr} 138$ 913 V15.0.0, p. 40, 2018.

[29] 3GPP, "TS 138 104-V15.2.0-5G; NR; Base Station (BS) radio transmission and reception (3GPP TS 38.104 version 15.2.0 Release 15)," Online Available, 2018.

[30] M. Fattah, M. Abdellaoui, D. Daghouj, S. Mazer, M. El Ghazi, M. El Bekkali et al., "Multi band OFDM alliance power line communication system," in Procedia Computer Science, vol. 151, 2019, pp. 1034-1039, doi: 10.1016/j.procs.2019.04.146.

[31] S. Ahmadi, "Link-Level and System-Level Performance of LTE-Advanced," LTE-Advanced, pp. 875-949, 2014, doi: 10.1016/b978-0-12-405162-1.00011-3.

[32] Z. Shi, L. Wang, S. Ma, G. Yang, and Y. Yao, "Goodput Maximization of HARQ-IR over Arbitrarily Correlated Rician Fading Channels,” IEEE Access, vol. 6, pp. 28903-28911, 2018, doi: 10.1109/ACCESS.2018.2839615.

[33] O. León, J. Hernández-Serrano, and M. Soriano, "Securing cognitive radio networks," International Journal of Communication Systems, vol. 23, no. 5, pp. 633-652, 2010, doi: 10.1002/dac.

[34] K. Norlund, T. Ottosson, and A. Brunstrom, "TCP fairness measures for scheduling algorithms in wireless networks," Second International Conference on Quality of Service in Heterogeneous Wired/Wireless Networks (QSHINE'05), vol. 2005, 2005, doi: 10.1109/QSHINE.2005.58.

[35] T. Hoßfeld, L. Skorin-Kapov, P. E. Heegaard, and M. Varela, "A new QoE fairness index for QoE management," 
Quality and User Experience, vol. 3, no. 1, pp. 1-23, 2018, doi: 10.1007/s41233-018-0017-x.

[36] U. Nwawelu, C. Ani, and A. Ahaneku, "Comparative Analysis of the Performance of Resource Allocation Algorithms in Long Term Evolution Networks," Nigerian Journal of Technology, vol. 36, no. 1, pp. 163-171, 2017, doi: 10.4314/njt.v36i1.21.

[37] A. Bin Sediq, R. H. Gohary, and H. Yanikomeroglu, "Optimal tradeoff between efficiency and Jain's fairness index in resource allocation," in IEEE International Symposium on Personal, Indoor and Mobile Radio Communications, PIMRC, 2012, pp. 577-583, doi: 10.1109/PIMRC.2012.6362851.

\section{BIOGRAPHIES OF AUTHORS}

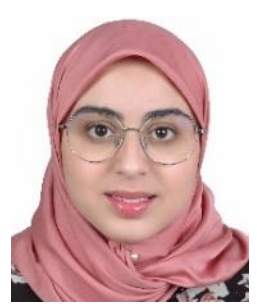

Asmae Mamane received her D.I. degree in networks and telecommunications from Hassan I University, Settat, Morocco in 2016. She is now a Ph.D. student in 5G Technology at Artificial Intelligence, Data Sciences, and Emerging Systems Laboratory of Sidi Mohamed Ben Abdellah University Fez Morocco. Her research interests include resource allocation techniques, scheduling algorithms, and improvement of QoS in 5G technology.

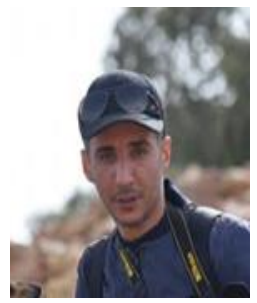

Mohammed Fattah received his Ph.D. in Telecommunications and CEM at the Sidi Mohamed Ben Abdellah University (USMBA), Fez, Morocco, 2011. He is a professor in the Electrical Engineering Department of the High school of technology at the Moulay Ismail University (UMI), Meknes, Morocco and he is responsible for the research team 'Intelligent Systems, Networks and Telecommunications', IMAGE laboratory, UMI.

Email: m.mohammedfattah@umi.ac.ma

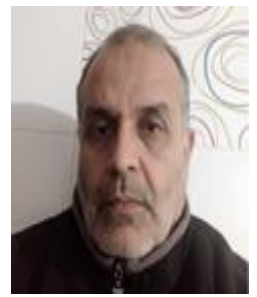

Mohammed El Ghazi is a professor in the Electrical and Computer Engineering Department of Superior School of Technology at the Sidi Mohamed Ben Abdellah University (USMBA), Fez, Morocco. $\mathrm{He}$ is a member of the Laboratory of Artificial Intelligence, Data Sciences and Emerging Systems.

Email: mohammed.elghazi@usmba.ac.ma

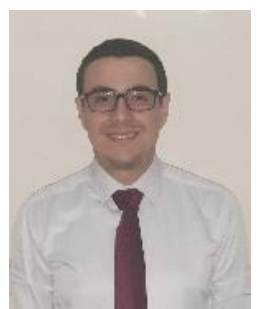

Younes Balboul is a professor in the National School of Applied Sciences at the Sidi Mohamed Ben Abdellah University (USMBA), Fez, Morocco. He is a member of the Laboratory of Artificial Intelligence, Data Sciences and Emerging Systems.

Email: younes.balboul@usmba.ac.ma

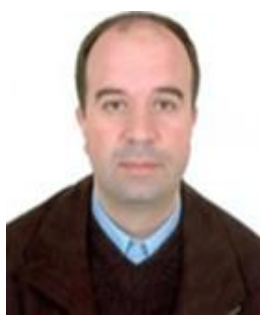

Moulhime El Bekkali is a professor in the National School of Applied Sciences at the Sidi Mohamed Ben Abdellah University (USMBA), Fez, Morocco. He is a member of the Laboratory of Artificial Intelligence, Data Sciences, and Emerging systems.

Email: moulhime.elbekkali@usmba.ac.ma

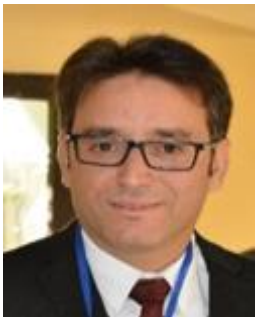

Said Mazer born in 1978. He received the Ph.D. degree in electronics and signal processing from the University of Marne-La-Vallée, Champs-sur Marne, France. He is currently a full Professor with the National School of Applied Sciences of Fez, Morocco. He is member of IASSE Laboratory, University of Sidi Mohamed Ben Abdellah Fez. His research interests include the development of microwave-photonics devices for radio-over fibre and wireless applications and he is also involved in network securitys.

Email: said.mazer@usmba.ac.ma 